\title{
Predicting Gynecological Malignancy at Surgery: Magic 8 Ball, or Paradigm Shift?
}

\author{
Hector O Chapa* \\ Medical Director, Women's Specialty Ctr, Dallas, Minimally invasive gynecologic surgery, USA
}

*Corresponding author: Hector O Chapa, Medical Director, Women's Specialty Ctr, Dallas, Minimally invasive gynecologic surgery, USA, Tel: +1 214-841-9222; E-mail: ChapaMD@Gmail.com

Rec date: Mar 12, 2014, Acc date: Apr 29, 2014, Pub date: May 01, 2014

Copyright: @ 2014 Chopr O H. This is an open-access article distributed under the terms of the Creative Commons Attribution License, which permits unrestricted use, distribution, and reproduction in any medium, provided the original author and source are credited.

\begin{abstract}
In medical school, future physicians are taught the phrase "primum non nocere", first do no harm. That adage motivates every physician, every healthcare provider, to go to great lengths to avoid an incorrect diagnosis for a patient. As a gynecological surgeon, that adage is put to the test with every operative patient dealing with a pelvic mass. Encountering an undiagnosed malignancy during and anticipated benign surgical intervention is stressful for the surgeon, but devastating to the unprepared patient. Recently, attention has focused on uterine morcellation at time of laparoscopic hysterectomy. Stemming from the inadvertent morcellation of a leiomyosarcoma in a patient presumed to have been benign fibroids, the question has arisen of informed consent disclosures versus the appropriateness of a selected surgery when the possibility of a malignancy exists (WSJ Dec 2013).
\end{abstract}

Keywords Pelvic masses; Uterine morcellation; Fibroids; Malignant leiomyomas; Multivariate assay; Ova1

\section{Introduction}

In medical school, future physicians are taught the phrase "primum non nocere", first do no harm. That adage motivates every physician, every healthcare provider, to go to great lengths to avoid an incorrect diagnosis for a patient. As a gynecological surgeon, that adage is put to the test with every operative patient dealing with a pelvic masswhether uterine or ovarian. Encountering an undiagnosed malignancy during an anticipated benign surgical intervention is stressful for the surgeon, but devastating to the unprepared patient.

Recently, attention has focused on uterine morcellation at time of laparoscopic hysterectomy. Stemming from the inadvertent morcellation of a Leiomyosarcoma (LMS) in a patient presumed to have been benign fibroids, the question has arisen of proper informed consent disclosures versus the appropriateness of a selected surgery when the possibility of a malignancy exists [1].

In 2009, the American College of Obstetricians and Gynecologists released their Committee Opinion (\#439) on the informed consent process [2]. As stated within its text, "the obligation to provide adequate information to a patient implies an obligation for physicians to be current in their own knowledge... about treatments and disease processes". Adequate disclosure must include patient awareness of possible risk of malignancy in the setting of any pelvic mass.

\section{In Perspective}

Fibroids are the most common type of tumor found in the female reproductive system. Fibroids have an estimated lifetime risk of $70 \%$ in Caucasian women and $80 \%$ in African-American women [3]. Alternatively, uterine sarcoma is rare occurring at a rate of 3 to 7 per 100,000 in United States [4].
In November 2012, a published study from Brigham and Women's Hospital published a review of 1091 instances of uterine morcellation at the hospital. The rate of unexpected sarcoma after the laparoscopic morcellation procedure was $0.09 \%$, nine fold higher than the rate currently accepted. These authors also concluded, "While, additional studies are warranted, these data suggest you and morcellation carry the risk of disseminating unexpected malignancy" with an increase in mortality than previously appreciated [5].

Additionally, the society of gynecologic oncology released a statement dated December 2013 under the topic "Morcellation". In the statement, "the SGO recognizes that currently there is no reliable method to differentiate benign from malignant leiomyomas before removal. Furthermore, these diseases often extremely poor prognosis even when specimens are removed intact" [6]. And therein lays the problem. While certain clinical factors may suggest a LMS (rapidly expanding mass, or a large solidarity mass), no reliable or definitive preoperative tool for diagnosis of this condition currently exist.

\section{Evaluation of the Uterine Mass}

Traditionally, the evaluation of a suspected pelvic mass commences with transvaginal/pelvic ultrasonography. Numerous authors have proposed various tools and techniques to improve ultrasonography's ability to differentiate a benign from a malignant smooth muscle mass.

Exacoustos et al. [7] compared gray-scale and color Doppler sonography for ability to differentiate between uterine LMS and leiomyoma [7]. The preoperative gray-scale and color Doppler sonographic findings of 8 patients with LMS, 21 patients with cellular leiomyomas, and 3 patients with smooth muscle tumors of uncertain malignant potential were obtained and compared with images of 225 patients with benign leiomyomas. All patients underwent myomectomy or hysterectomy. Sonographic characteristics were correlated to the histologic findings from surgery. LMSs were significantly larger than other uterine smooth muscle tumors. They 
were all solitary, and $7 / 8$ lesions had a diameter $>$ or $=8 \mathrm{~cm}$. Degenerative cystic changes were observed in 4 lesions, and increased peripheral and central vascularity was demonstrated in 7 lesions. Sensitivity, specificity, and positive predictive value of increased central and peripheral vascularity in the diagnosis of LMS were $100 \%$, $86 \%$, and $19 \%$, respectively. Combining other sonographic findings with marked central vascularity, positive predictive value increased to $60 \%$, but sensitivity decreased to $75 \%$.

Uterine myoma size and location may affect detection ability by sonography. To investigate this further, researchers from the National Institute of Health published findings comparing Magnetic Resonance (MR) imaging with transvaginal Ultrasound (US) for fibroid burden in pre-hysterectomy, pre-menopausal patients [8]. Eighteen women undergoing hysterectomy for symptomatic fibroids underwent preoperative pelvic US and MR imaging. Resected fibroids were correlated with the images.

In this study, the sensitivity of MR imaging was two-fold greater than US for the detection of uterine fibroids (MR imaging: $80 \%$; US: $40 \%$ ) using pathological specimens as a gold standard. However, when fibroids were identified surgically, the positive predictive value for MR imaging and US were similar. Even after excluding small fibroids (diameter equivalence: $\leq 1 \mathrm{~cm}$ ) from consideration, the sensitivity of US remained low (47\%). This observation suggests that MR imaging be considered as the best modality for the detection of uterine fibroidsat least in clinical research- especially considering its superior ability to detect smaller lesions.

Other published reports have suggested that MRI may be helpful in women with suspicion of a uterus sarcoma. However no definitive diagnosis can be made. High signal intensity cannot be considered a reliable indicator of uterine sarcoma [9].

\section{Preoperative Biopsy}

As part of routine preoperative workup for patients with fibroids and typical abnormal uterine bleeding, endometrial sampling has been evaluated as a preoperative tool to rule out uterine sarcoma. Unfortunately, the sensitivity of Endometrial Biopsy (EMB) for this has been limited. Reports have documented 33\% -67\% sensitivity for sarcoma pathology by EMB. [10,11]. It is important to note, however, that in these cited referenences no distinction was made between leiomyosarcoma and endometrial stromal sarcoma. Endometrial biopsy should be more useful to distinguish between endometrial stromal sarcoma and epithelial tumors than to identify leiomyosarcoma that is usually arising from the myometrial layer.

With these limited abilities to preoperatively diagnose uterine sarcoma, it seems that the magic 8 ball will likely land on a "benign" likelihood simply based on disease prevalence.

Though devastating in its occurrence, the absolute number of this disease process is thankfully still low. It is still the position of the ACOG that morcellation is a benefit and option as a minimally invasive hysterectomy for the vast majority of women [12].

\section{Evaluation of Ovarian Masses}

While much attention has focused on uterine sarcomas recently, as gynecologist we cannot forget a disease process which is significantly more frequent in the population: ovarian cancer. A women's lifetime risk of ovarian cancer is 1 in 70 . Ovarian cancer still remains among the deadliest of gynecologic malignancies. When deciding on the type of surgery for a patient with an adnexal mass, estimating the risk of malignancy is essential. Once again, the question arises of ability for better preoperative diagnosis (risk stratification) before the index surgery. This is critically important since it is well recognized and accepted that the patient's best chance for survival - after diagnosis of ovarian cancer- is having optimal staging and debulking at that first index surgery by a trained physician. Both the American College of obstetricians and gynecologist and Society of Gynecologic Oncology support referral to a gynecologic oncologist when an ovarian cancer is suspected [13].

For years, though, gynecologists had imperfect means in predicting which ovarian masses were more likely to be a malignancy. Here, the magic 8 ball often came to play:

The staple of ovarian mass diagnosis - the ultrasound - is a valuable tool in the diagnosis of ovarian pathology. As with uterine masses, investigators have proposed various algorithms to improve ultrasonography's discretion for benign vs malignant ovarian neoplasms. Several studies have shown that the risk of malignancy is very low in unilocular ovarian cysts $[14,15]$. Timmerman et al. [16] evaluated an adoptable strategy for ultrasound risk stratification of the ovarian mass [16]. The design was to prospectively assess the diagnostic performance of simple ultrasound rules to predict benignity/malignancy in an adnexal mass, using histological classification of the excised adnexal mass as benign or malignant as the reference standard.

\section{As described within their publication:}

"The rules comprised five ultrasonic features (including shape, size, solidity, and results of colour Doppler examination) to predict a malignant tumour ( $\mathrm{M}$ features) and five to predict a benign tumour ( $\mathrm{B}$ features). If one or more $M$ features were present in the absence of a $B$ feature, the mass was classified as malignant. If one or more B features were present in the absence of an $\mathrm{M}$ feature, it was classified as benign. If both $\mathrm{M}$ features and $\mathrm{B}$ features were present, or if none of the features was present, the simple rules were inconclusive" [16].

A total of 1938 women with an adnexal mass were examined with ultrasound by the principal investigator at each center (19 sites, 8 countries) with a standardized research protocol. Of the 1938 patients with an adnexal mass, $72 \%$ had benign tumors, $19.2 \%$ had primary invasive tumors, $5.7 \%$ had borderline malignant tumors, and $3 \%$ had metastatic tumors in the ovary. The simple rules yielded a conclusive result in 1501 (77\%) masses, for which they resulted in a sensitivity of $92 \%$ (95\% confidence interval $89 \%$ to $94 \%$ ) and a specificity of $96 \%$ ( $94 \%$ to $97 \%$ ). The corresponding sensitivity and specificity of subjective assessment were $91 \%$ ( $88 \%$ to $94 \%$ ) and $96 \%$ (94\% to $97 \%$ ).

The authors concluded that this simplified classification was the most predictive of ovarian mass status, even when compared to other algorithms (the risk of malignancy index, and two ultrasound regression models).

Despite this advance in imaging interpretation once a mass is identified, ultrasound has not shown promise as an ovarian mass screening tool. The Prostate, Lung, Colorectal, Ovarian Study was a randomized controlled trial evaluating the use of annual CA-125 levels along with screening ultrasound. 78,216 women were enrolled between 1993 and 2010. 33\% of women with false positive results underwent surgery in the cohort, with $15 \%$ major complication rate in this group of women [17]. 
Page 3 of 4

\section{Serum Biomarkers}

The serum test CA125 (first and second generation) has often been viewed as the "ovarian triage test of choice". However, it is not representative of all histologies of ovarian cancers nor is it helpful for early-stage disease [18].

Then, in 2009, the FDA approved the first serum proteomic multivariate assay (MVA) for ovarian mass triage and referral (Oval Vermillion, Inc. Austin, TX, USA). This bioassay uses 5 serum biomarkers to generate a calculated risk score for cancer prediction, based upon the patient's menopausal status. Compared with the traditional evaluation of CA125, this multivariate detected $76 \%$ of cancers missed by CA125 (second generation), including all advanced stage malignancies, with $99 \%$ overall sensitivity for epithelial ovarian cancer, and $91 \%$ sensitivity for other histology types [19].

In January 2014, Longoria et al. [20] analyzed the effectiveness of this MVA in detecting early-stage ovarian malignancy compared to clinical assessment, CA 125-11, and modified ACOG guidelines for woman undergoing surgery for an adnexal mass. The authors concluded after analysis of 1016 women that this diagnostic assay combined with clinical assessment demonstrated higher sensitivity for early-stage ovarian malignancy compared to clinical assessment alone, CA 125-11, and even the modified ACOG guidelines. This was true across menopausal status [20].

In February 2014, Goodrich et al. [21] reinforced the synergy of imaging ultrasound with multivariate assay serum testing in this setting [21]. Subjects were recruited in 2 related prospective, multiinstitutional trials that involved 44 sites across the United States. Women had ovarian imaging, biomarker analysis, and surgery for an adnexal mass. Of the 1110 women who were enrolled with an adnexal mass on imaging, 1024 cases were evaluable. There were 255 malignant and 769 benign tumors. When more than one test was applied (MVA and/or ultrasonography), performance was influenced by how the tests were combined: The parallel ("or") combination of OVA1 and imaging results in high sensitivity (98\%) and high negative predictive value (98\%), while the serial ("and") combination of OVA1 and imaging (AND) improves specificity (75\%) and positive predictive value $(48 \%)$.

Improved ability of ovarian mass cancer prediction represents a leap forward for best clinical practice for these patients. Future generations of Oval may seek an expanded indication to include triage of patients into surgical or non-surgical categories. That would represent yet another evolution in the clinical pathway of adnexal mass management.

The Society of Gynecologic Oncology published their statement of acknowledgement of Oval multivariate assay in June 2013. It is available on the SGO website SGO.org [22]

For this most deadly of gynecologic malignancies (ovarian cancer), gynecologists may finally have an opportunity to put away the magic 8 ball in leu of a new paradigm. Serum Multivariate Analysis allows for improved ovarian cancer detection, earlier referral, and better patient care allowing for the best surgery choice for the appropriate condition.

\section{Closing Remarks}

The prediction of gynecologic malignancy for pelvic masses does not have to be a "magic 8 ball" exercise. Evidence based algorithms and improved diagnostic aids for surgical triage can help guide physicians in patient management. By staying current in the literature and practicing true patient informed consent, we can strive for better clinical outcomes. Nonetheless, however, despite our best intentions and techniques, we must grapple with the reality that certain unanticipated findings and events may occur. As stated by one of my Attending Physicians during my residency training," Never voluntarily invite HARM to the treatment plan, but always anticipate him crashing the party".

Ova1 Indications (per manufacturer):

Patient with diagnosed ovarian mass not yet referred to a Gynecologic oncologist

Patient scheduled for surgery

Age greater than 18

No prior history of cancer in the last 5 years

Patients with normal Rheumatoid Factor, and Triglycerides levels $<4.5 \mathrm{~g} / \mathrm{L}$

Oval is not currently indicated for ovarian cancer screening.

\section{Disclosures}

Dr Hector O. Chapa serves as a medical consultant and research consultant to Vermillion, Inc., manufacturer of Ova1. No financial or corporate assistance was provided for the formulation of this commentary.

\section{References}

1. Levitz J (2013) Doctors eye cancer risk in uterine procedure. WSJ Dec 18, 2013.

2. The American Congress of Obstetricians and Gynecologists (2009) Informed Consent. Committee Opinion \#439.

3. Baird DD, Dunson DB, Hill MC, Cousins D, Schectman JM (2003) High cumulative incidence of uterine leiomyoma in black and white women: ultrasound evidence. Am J Obstet Gynecol 188: 100-107.

4. Brooks SE, Zhan M, Cote T, Baquet CR (2004) Surveillance, epidemiology, and end results analysis of 2677 cases of uterine sarcoma 1989-1999. Gynecol Oncol 93: 204-208.

5. Seidman MA, Oduyebo T, Muto MG, Crum CP, Nucci MR, et al. (2012) Peritoneal dissemination complicating morcellation of uterine mesenchymal neoplasms. PLoS One 7: e50058.

6. https://www.sgo.org/newsroom/position-statements-2/morcellation/

7. Exacoustos C, Romanini ME, Amadio A, Amoroso C, Szabolcs B, et al. (2007) Can gray-scale and color Doppler sonography differentiate between uterine leiomyosarcoma and leiomyoma? J Clin Ultrasound 35: 449-457.

8. Levens ED, Wesley R, Premkumar A, Blocker W, Nieman LK (2009) Magnetic resonance imaging and transvaginal ultrasound for determining fibroid burden: implications for research and clinical care. Am J Obstet Gynecol 200: 537.

9. Amant F, Coosemans A, Debiec-Rychter M, Timmerman D, Vergote I (2009) Clinical management of uterine sarcomas. Lancet Oncol 10: 1188-1198.

10. Leibsohn S, d'Ablaing G, Mishell DR Jr, Schlaerth JB (1990) Leiomyosarcoma in a series of hysterectomies performed for presumed uterine leiomyomas. Am J Obstet Gynecol 162: 968-974.

11. Bansal N, Herzog TJ, Burke W, Cohen CJ, Wright JD (2008) The utility of preoperative endometrial sampling for the detection of uterine sarcomas. Gynecol Oncol 110: 43-48. 
Citation: Chapa OH (2014) Predicting Gynecological Malignancy at Surgery: Magic 8 Ball, or Paradigm Shift?. J Clin Case Rep S1: 009. doi: 10.4172/2165-7920.S1-009

Page 4 of 4

12. (2009) The American Congress of Obstetricians and Gynecologists. Choosing the right of hysterectomy for benign disease. ACOG committee opinion number 444.

13. American College of Obstetricians and Gynecologists Committee on Gynecologic Practice (2011) Committee Opinion No. 477: the role of the obstetrician-gynecologist in the early detection of epithelial ovarian cancer. Obstet Gynecol 117: 742-746.

14. Granberg S, Wikland M, Jansson I (1989) Macroscopic characterization of ovarian tumors and the relation to the histological diagnosis: criteria to be used for ultrasound evaluation. Gynecol Oncol 35: 139-144.

15. Roman LD (1998) Small cystic pelvic masses in older women: is surgical removal necessary? Gynecol Oncol 69: 1-2.

16. Timmerman D, Ameye L, Fischerova D, Epstein E, Melis GB, et al. (2010) Simple ultrasound rules to distinguish between benign and malignant adnexal masses before surgery: prospective validation by IOTA group. BMJ 341: c6839.

17. Buys SS, Partridge E, Black A, Johnson CC, Lamerato L, et al. (2011) Effect of screening on ovarian cancer mortality: the Prostate, Lung,
Colorectal and Ovarian (PLCO) Cancer Screening Randomized Controlled Trial. JAMA 305: 2295-2303.

18. Moss EL, Hollingworth J, Reynolds TM (2005) The role of CA125 in clinical practice. J Clin Pathol 58: 308-312.

19. Ueland FR, Desimone CP, Seamon LG, Miller RA, Goodrich S, et al. (2011) Effectiveness of a multivariate index assay in the preoperative assessment of ovarian tumors. Obstet Gynecol 117: 1289-1297.

20. Longoria TC, Ueland FR2, Zhang Z3, Chan DW3, Smith A4, et al. (2014) Clinical performance of a multivariate index assay for detecting earlystage ovarian cancer. Am J Obstet Gynecol 210: 78.

21. Goodrich ST, Bristow RE, Santoso JT, et al (2014) The effect of ovarian imaging on the clinical interpretation of a multivariate index assay. Epub. Am J Obstet Gynecol.

22. https://www.sgo.org/newsroom/position-statements-2/multiplex-serumtesting-for-women-with-pelvic-mass/
This article was originally published in a special issue, entitled: "Obstetrics and Gynecology", Edited by Yigit Cakiroglu Department of Obstetrics and Gynecology Kocaeli University Kocaeli, Turkey 